\title{
Programa de alfabetización económica sobre conductas de emprendimiento en pobladores quechua hablantes de caseríos de Caraz
}

\author{
Economic literacy program on entrepreneurship behaviors in \\ quechua speakers rural landscapes residents, Caraz \\ Mildred Paredes T11., Héctor Hernández V., Emma Campos P., Luís Vicuña P., Juan Merino M., \\ Juan Morocho S., María Rosario Quesada M. \\ Universidad Nacional Mayor de San Marcos, Lima, Perú \\ (RECIBIDO 15-08-2014, AcEPTADO 20-10-2014)
}

\begin{abstract}
RESUMEN
Se estudió la efectividad del programa de alfabetización económica en el desarrollo de conductas de emprendimiento en pobladores quechua hablantes de Caraz, Ancash. La alfabetización económica concebida como estrategia válida en la transmisión de saberes intergeneracionales para la formación de conceptos básicos de economía aplicados a la gestión de unidades productivas. Se empleó un diseño cuasiexperimental con pre y postprueba, y cualitativa en torno al imaginario colectivo. El programa de alfabetización económica, variable independiente, y las conductas de emprendimiento, variable dependiente; observadas en la muestra del grupo experimental constituida por colaboradores del Caserío Queral y grupo de control, padres de familia del 3er grado de la I. E. 2 de Mayo de Caraz. Se aplicó: Cuestionario de conocimiento de saberes, Registro de Observación de Conductas de Emprendimiento (ROCE-2010) y la Escala de Actitudes hacia el Emprendimiento (EAE-2010 Confiabilidad de 0,83). Mediante estadísticos descriptivos e inferenciales no paramétricos se analizaron la data. Se encontró que El programa de alfabetización económica afecta las conductas de emprendimiento, expresa asimismo en la formación de una unidad productiva que tuvo una producción comercializada y reinvertida de un producto de su expresión cultural (UNESCO, 2005).
\end{abstract}

Palabras clave: Alfabetización económica, emprendimiento, percepción económica, actitudes hacia el emprendimiento, transmisión intergeneracional, producto cultural.

1 Docente Facultad de Psicología UNMSM. E-mail: mildredparedestarazona@gmail.com 


\begin{abstract}
The effectiveness of economic literacy program in the development of entrepreneurial behaviors in Quechua speaking people Caraz, Ancash was studied. Economic literacy conceived as a valid strategy in the intergenerational transmission of knowledge to the formation of basic economics applied to the management of production units. A quasi-experimental pre and post-test, and qualitative around collective imaginary design was used. Economic Literacy Program, independent variable, and entrepreneurial behavior, dependent variable; observed in the sample of the experimental group consisting of rural landscape residents collaborators Queral and control group, parents of 3rd grade of IE 2 de Mayo, Caraz. Was applied: Questionnaire knowledge of knowledge, Behavior Observation Record Entrepreneurship (ROCE-2010) and the Scale of Attitudes Toward Entrepreneurship (EAE-2010 Reliability 0.83). Descriptive statistical and inferential the nonparametric data were analyzed. It was found that the Program of Economic Literacy affects behaviors Entrepreneurship, also expressed in the formation of a productive unit that had a commercialized and reinvested a product of their cultural expression (UNESCO, 2005) production.
\end{abstract}

Keywords: Economic literacy, entrepreneurship, economic perceptions, attitudes toward entrepreneurship, intergenerational transmission, cultural product.

\title{
INTRODUCCIÓN
}

La situación de postergación y atraso de la población rural en el Perú se mantiene hasta el momento como un mal endémico por el limitado acceso a conocimientos, tecnologías, infraestructura y capital; propiciando en dicho contexto socio-económico el aprendizaje de conductas dependientes y a la espera de la generosidad o filantropía de otros. La disposición a la ayuda externa "por la condición de pobre" y a la ínfima participación en el mercado con actividades de emprendimiento, como también se acceden a trabajos, son a los que requieren poca preparación y la compensación por el trabajo dependiente no llega al sueldo mínimo, generando la creencia de impotencia para superar o modificar su status quo.

La sobreprotección debilita la personalidad y propicia una situación no sostenible económicamente, de ahí que la cooperación o programas sociales que en lugar de ser de ayuda para el objetivo de proporcionar bienestar, más bien son un factor de alto riesgo, conducente a la anomia, la adopción de comportamientos de riesgo (ej. embarazo adolecente), el abandono del campo y la desintegración de la comunidad, repercutiendo en las familias y acrecentando el circuito de exclusión y reforzando comportamientos de dependencia.

Sin embargo, a pesar de dichas condiciones en dicho contexto socio económico cultural, la población manifiesta el potencial y riqueza de su capital social o humano, evidenciado en un crecimiento del $5 \%$ en los últimos cinco años "gracias al impulso propio de sus pobladores" a partir de la construcción de carreteras en zonas rurales, Webb (2012). La valoración y el reconocimiento del propio potencial con la perspectiva del desarrollo de competencias, demanda crear espacios con estrategias para instrumentar los principios de aprendizaje en la formación de conceptos 
básicos de economía aplicados a la gestión de unidades productivas, proponiendo un programa de alfabetización económica en conductas de emprendimiento en pobladores quechua hablantes para la transmisión de saberes intergeneracionales.

Investigación aplicada, con diseño cuasiexperimental, medición antes y después de la intervención, complementándose con el análisis cualitativo del proceso grupal de los talleres de capacitación a propósito de observar conductas de emprendimiento en las colaboradoras quechua hablantes de Caraz.

El grupo de colaboradoras adultas del Caserío de Queral, se constituyo en el grupo cuasiexperimental y el grupo de control provenientes de la zona urbana geográfica de intervención-también quechua hablante-. El programa de capacitación diseñado para la alfabetización en términos de economía básica son con temas propios del ámbito de la gestión de unidades productivas y según las dimensiones del emprendimiento, programadas en sesiones de acorde al modo de vida de la comunidad, transacciones económicas, organización comunal y cultura.

Cada sesión de capacitación siguió la modalidad de taller, aplicándose dinámicas grupales y técnicas para facilitar la participación. La temática de cada taller tuvo como eje central la naturaleza de sus actividades económicas relacionadas con los bienes culturales identificadas en el estudio de Paredes, Hernández, Vicuña, Rivera y Arias (2009), y para la etapa de producción del proceso de la dinámica de grupo, se incorporaron tareas laborales propias de su que hacer.

Las tareas ejecutadas a partir de sus saberes y habilidades demostradas en la elaboración de la chalina, producto cultural de la comunidad, con potencial de ser comercializados en concordancia a lo prescrito por la Unesco (2005), -está en vía de rescate y elaboración pero que aún no se ha constituido en un proyecto productivo de la comunidad-, permitió a las colaboradoras identificar los conceptos básicos de economía y de gestión, durante la realización de tareas del proceso de manufactura de la chalina, manejo de insumos, modo de organización del trabajo y comercialización.

La decisión de las colaboradoras de elegir las chalinas, entre otros productos textiles, se debió a que era un producto que culturalmente solo realizan las mujeres, en cambio el telar en sus diversas modalidades y la costura de ropa son actividades propias de los varones.

Etnografía del caserío de Queral, está situado al sur de Caraz en las estribaciones de la Cordillera Blanca en dirección opuesta al nevado principal de la provincia, el Huandoy, a una altura de 3200msnm. Apenas cuenta con los servicios básicos, sin desagüe. Los pobladores son agricultores en su mayoría y uno que otro varón eventualmente se dedica al tejido, mediante el telar de cintura, utilizado para hacer mantas, entre sus productos manufacturados.

El nivel de instrucción de los adultos llega al nivel técnico en los varones y es más elevado que en las mujeres. Las familias suelen tener un promedio de 4 hijos, generalmente inician su escolaridad en el I.E. del Caserío, desde la educación 
inicial al 6to. Grado para continuar en Pampacocha o en la ciudad de Caraz u otro distrito de la provincia, el mayor numero abandono y con el mayor número de iletradas son de las mujeres que los varones.

La actividad económica principal es la siembra de alverja como producto comercial y en segundo lugar la papa; producto para el autosostenimiento, cooperación y reciprocidad en tiempos de crisis; hay costumbre de hacer aprovisionamiento para tiempos difíciles, incluyendo el trigo, cebada y maíz si tuvieron buena (adecuada) cosecha.

Los comuneros eligen una junta directiva, la que convoca y canaliza las decisiones tomadas en asamblea. Es el órgano de gobierno, responsable de autorizar la intervención en la comunidad y suscribir acuerdos que debe quedar registrado en acta, con firma o una cruz o huella digital, condición para desarrollar la investigación.

En este contexto se aplicó un programa de alfabetización económica que incrementó significativamente las conductas de emprendimiento en pobladores quechua hablantes de caseríos de Caraz. Entre las conductas significativas al término del Programa se evidenció la conducta de emprendimiento a través de la organización de una unidad productiva denominada "Asociación de productores del caserío de Queral Tuco Wain Perú"; un resultado a favor de la hipótesis.

\section{Planteamiento del problema}

En una sociedad en la que el trabajo dependiente escasea, el emprendimiento se convierte en un recurso vital, entendiéndose como una respuesta efectiva a partir de la iniciativa y la perseverancia para desarrollar actividades económicas independientes, sean productivas o de servicios, movilizando capital, competencias y aptitudes en experticia al dedicarse como un trabajo, y generando empleo con el competente de innovación o creatividad. Obteniendo en principio el autosostenimiento para el caso de la Mypes, con visión y misión de sostenibilidad. El resultado del emprendimiento tendrá mayor efecto en el conjunto de la sociedad, si logra producir el efecto multiplicador al generar un círculo virtuoso en el cual la capacidad adquirida genere una mayor productividad, en especial empleo y satisfacción al desempeñar un trabajo descrito como de calidad.

En la zona de estudio se observa la carencia del trabajo compensado apropiadamente - menos equitativo-, no hay un justo comercio, condición que sume en la pobreza extrema a sus habitantes, traslada de padres a hijos, aunado a que entre sus actividades, la agricultura, resulta insuficiente para alcanzar niveles de calidad de vida aceptables, pese a ello, al tener con que laborar (con que comprar abono), el trabajo en sí, es una motivación intrínseca. Razón entre otras, justifica revertir está secuencia negativa con productividad y crecimiento económico que aúne su desarrollo personal. Donde la variable dependiente, conducta de emprendimiento es un indicador de evaluación al término de la aplicación del programa.

La socialización económica, promovida en la Universidad de la Frontera, Chile por Marianela Denegri, entraña conceptos y recursos necesarios que la convierte 
en una valiosa alternativa de instrumentación para lograr emprendimiento en las poblaciones más diversas, en independencia de su nivel de instrucción, mediante el programa de alfabetización económica realizada según el grupo etario, medida como conducta y actitud al emprendimiento.

El planteamiento del problema enmarcado en la realidad de la población de las zonas alto andina que cíclicamente se mantienen socioeconómicamente excluidas, de ahí que, la socialización económica sea una alternativa para el desarrollo de competencias que lleven al emprendimiento. Emprender es la acción para generar asociaciones productivas capaces de generar beneficios para los asociados, iniciando con la alfabetización económica que proporcione conocimiento base para el diseño de estrategias o plantear políticas sociales que encamine de manera sostenible el progreso de su situación, en concordancia a su propia decisión y cultura. Pese a la manifiesta utilidad económica y social, la confección de chalinas no son consideradas como generadores de ingresos y aun menos como una actividad económica productiva sostenible, planteándose la necesidad de observar al aplicar la variable del programa de alfabetización en términos básicos de gestión si logra potenciar el emprendimiento, se plantea ¿Es eficaz el Programa de Alfabetización Económica sobre Conductas de Emprendimiento en Pobladores Quechua Hablantes de Caseríos de Caraz?

\section{Referencias teóricas}

La educación económica trata de la "Acción educativa intencionada cuyo objetivo es facilitar que niños y adultos construyan nociones económicas básicas y desarrollen estrategias para tomar decisiones adecuadas que les permitan situarse ante la sociedad de consumo como personas conscientes, críticos, responsables y solidarias, la que se traduce en alfabetización económica" Denegri et al (1999). Esta acción efectiva planificada en la comunidad campesina Cruz de Mayo, en sus caseríos de influencia de Caraz, posibilitaría la transferencia de saberes de manera intergeneracional mediante "el aprendizaje social que significa transferir patrones culturales, actitudes en una perspectiva general de vida" Ardila (1993), en el contexto de su propia cultura para el desarrollo de la actividad económica. Se sabe que el conocimiento de toda cultura se construye, dinamismo que es continuo con el aprendizaje, legado fortalecido por la transmisión intergeneracional, explicado también con las conclusiones de Taberna y Peralta (2009), que priorizan el peso tanto de las teorías asociacionistas, del conocimiento nuclear, marcadores conceptuales y razonamiento por analogías. Las mismas que contemplan la importancia del contexto sociocultural, aún cuando cada una establece mecanismos de cambio cognitivo.

Dichas aproximaciones centradas en una visión sociocultural ponen en relieve que las formas cognitivas, así como la formación de conceptos, dependen, derivan y son constituidas en el marco de un contexto interpersonal, y no solo por los esfuerzos individuales que los niños puedan realizar para conocer su mundo. Es decir, al no tener el niño el paradigma de los mayores que respalden o fortalezcan el apren- 
dizaje con estímulos secundarios, el subdesarrollo quedará contextualizado. Lo mismo ocurre con el logro del nivel requerido para la realización de inferencias. Piaget, (1971, p. 80), afirma que "en muchas sociedades el pensamiento adulto no rebasa el nivel de las operaciones concretas y, por consiguiente, no llega al de las operaciones operacionales formales -entre los 12 y los 15 años-" De ahí, aun cuando no es el objetivo el desarrollo cognitivo de los adultos de la comunidad, se hace necesario proporcionar información que les posibilite desarrollar su conocimiento para optar conceptos y evitar el rechazo de aquellas oportunidades sustentadas en sus propias habilidades que puedan iniciar sus niños, base sociocultural fundamental para el aprendizaje.

Las investigaciones en la zona señalan que las actitudes hacia el empoderamiento mediante unidades productivas son positivas y difieren según sexo, edad y condición (puesto o miembro de la comunidad), Paredes et al., (2009b). Hay relación entre el conocimiento de la demanda y formar articulaciones económicas, Paredes y col, (2007). Conocimiento igualmente motivador para fortalecer las iniciativas para involucrarse en programas de capacitación. El Caserío, se constituye una unidad productiva en la articulación empresarial u organizacional, siendo Queral un caserío de la comunidad campesina Cruz de Mayo, Paredes, (2009), Paredes y De Miguel, (2004). Las colaboradoras de Queral eligieron y percibieron las Chalinas entre las expresiones culturales y le atribuyeron como productos comerciales Paredes y Col. (2009). Producto para lograr crear unidades económicas a propósito de reducir la brecha de desigualdad en la distribución (PNUD, 2010) y ser una alternativa viable de producción sostenible y enriquecedora para el desarrollo de dicha población.

Al conocer el desarrollo cognitivo según la edad cronológica de los escolares colaboradores de la zona de estudio, que debe ser estimulado para facilitar la adquisición de las nociones básicas de economía, nos alerta, al ser uno de los indicadores significativos para el proceso de socialización económica, que se deben tenerse en cuenta el emprendimiento del caserío como unidad económica de la articulación organizacional de la comunidad campesina Cruz de Mayo, Caraz.

La Comunidad al ser un espacio sociocultural sumamente complejo y delicado, tanto por las sensibilidades, las percepciones y los imaginarios, evidenciando su diversidad y solidez en su conducta al tomar decisiones, en especial aquellas por acuerdo en asamblea, exige de los investigadores entender su lógica para interactuar de manera responsable e interdependiente, distendida y en confianza, requisitos necesarios para la transferencia de conceptos económicos o intervención, pues "en realidad las personas no se resisten al cambio, se resisten a que se les impongan el cambio" Bregman, P. (CEO / 2009).

Sabiendo que las redes sociales, en especial las de parentesco, son una fortaleza que tienen los miembros de la comunidad para enfrentarse a las exigencias de su entorno; también existe la debilidad de la desconfianza para participar en negocios en forma asociada. Las redes sociales pueden explicar lo que es importante en 
la concepción de rentabilidad está mayormente hacia el soporte social o parental que en el establecimiento de un negocio por ganancia pecuniaria para los comuneros. Ante ésta norma de conducta sustentada en sus valores culturales, orienta a encontrar otras alternativas desde la psicología organizacional y otras ciencias o disciplinas a contribuir a la solución de problemas socio-económicos a través del emprendimiento en zonas rurales.

El proceso de aprendizaje vivencial, proporciona el marco para diseñar talleres en base a la realización de las tareas y funciones propias de su organización laboral para la elaboración y comercialización de la chalina. Se dio una etapa previa de identificación y rescate de sus expresiones culturales en productos comerciales, Paredes y Colb. (2009) en el proceso de intervención, para que las colaboradoras finalmente identificaran y seleccionaran a la chalina entre sus productos culturales.

En síntesis, se busca contrastar la hipótesis: El programa de alfabetización económica incrementa significativamente las conductas de emprendimiento en pobladores quechua hablantes de caseríos de Caraz.

\section{Método}

La población está constituida por mujeres mayores de 16 años quechua hablantes del caserío de Queral de la comunidad campesina Cruz de Mayo, de influencia a Caraz. Caserío cuya población está formada en su mayor parte por comuneros quechua hablantes, identificado con menores recursos y con percepciones menos favorables respecto a sus condiciones de trabajo, Paredes y Cols. (2005) y la otra muestra de mujeres también quechua hablantes de la Zona urbana en Caraz.

Las colaboradoras estuvieron distribuidas entre el grupo experimental y el grupo de control. El primero fue integrado por 21 madres pobladoras del caserío Queral y el segundo por 22 madres de familia del 3er. Grado de la I.E. 2 de Mayo de Caraz, incorporadas a la investigación por su participación en un taller de capacitación para desarrollar una actividad productiva no tradicional (bisutería) para obtener fondos para su aula, sin aplicar la V.I. que era para la condición experimental.

El Programa de alfabetización económica (V.I.) contiene conceptos básicos de economía, transferidos mediante situaciones concretas e imágenes, percibidas durante la elaboración, comercialización y organización organizacional y la técnica de la dinámica de grupo observar la gestión del producto textil cultural - la chalina- a propósito de propiciar en las colaboradoras la revaloración cultural y económica de la producción de las expresiones culturales locales. En el grupo de control, no se impartió los términos económicos en la elaboración de bisutería, pero si sobre los aspectos técnicos solicitado en la necesidad de capacitación.

El programa de alfabetización económica aplicado al grupo cuasi experimental consta de 12 talleres: 1. Formación de la organización de la unidad económica productiva; 2 . Toma de decisión de ser miembro de la unidad económica productiva; 3. Formación del comité especializado decreto supremo 008-91-TR, el 
que está estructurado en: Comité de Directiva (Presidenta de Comisión); Comité de Producción, Comité de Tesorería (tesorero). Inicialmente se formó además el Comité de Capacitación pero fracaso, 4. Opción de organización de la unidad económica productiva: Asociación; 5. Elección del Nombre de la Asociación de la Comunidad Campesina Cruz de Mayo, Caraz: Asociación de Productores del Caserío de Queral Tuco Wain Perú; 6. Elección del símbolo para la "marca" en base de su imaginario; 7. Taller de producción; 8. Taller en Comercialización: Canal de Ventas, Feria Municipal; 9. Taller de Producción de una nueva línea de productos; 10. Taller de Costos; 11. Taller de Distribución de Recursos;12. Taller de Revaluación y retroalimentación.

El contenido de cada taller tiene por objetivo, utilizar los términos básicos de economía y de gestión para el emprendimiento, tales como: 1. Aporte de asociación, 2. Asociación, 3. Comisión, 4. Comités, 5. Compra, 6.Costos, 7. División de trabajo, 8. Envoltura, 9. Empaque, 10. Feria, 11. Insumos, 12. Línea de producción, 13. Logotipo, 14. Mano de obra, 15. Marca, 16. Materia prima, 17. organización, 18. Precio, 19. Producción, 20. Productos, 21. Promoción, 22. Venta.

La Conducta de emprendimiento (V.D.); es identificada mediante las siguientes dimensiones que la describen: Disposición a aprovechar la oportunidad, Aprovechamiento y responsabilidad del recurso económico y humano, Disposición al riesgo, Disposición a la innovación, Estabilidad y serenidad en incertidumbre, Administración del caos.

Participantes colaboradoras, entre ellas madres de familia, quechua hablantes del caserío de Queral y del 3er. Grado del IE 2 de Mayo de Caraz, es la variable de control.

Se aplicaron estadísticos descriptivos e inferenciales no paramétricos a los datos obtenidos mediante los instrumentos construidos para el presente estudio, además de hacer cálculo del tamaño del efecto en los grupos, experimental y de control.

\section{Técnicas e instrumentos}

Los instrumentos empleados son el cuestionario de conocimiento de saberes y la escala de actitudes hacia el emprendimiento. Así mismo, se empleó el Registro de Observación de Conductas de Emprendimiento (ROCE-2010), del tipo seriado y de flash. La técnica de la dinámica de grupo para la transmisión de conceptos de manera participativa y lúdica tiene por contenido la actividad laboral rescatada, cuyas tareas en acciones y actos son funciones propias de la manufactura de la chalina, por ende son medios que de manera práctica se facilitan relacionar con el concepto de la socialización económica.

Registros de observación de conductas de emprendimiento (ROCE- 2010).

El registro de observación de conductas, tiene la validez de contenido mediante el criterio de jueces, para esto se solicitó la apreciación de cinco jueces quienes opinaron sobre la pertinencia de los reactivos. Para la fiabilidad de las puntuaciones 
Mildred Paredes T., Héctor Hernández V., Emma Campos P., luís Vicuña P., Juan Merino M., Juan Morocho S.

del test de conductas se hizo un análisis de consistencia interna, obteniéndose un coeficiente $\alpha=.90$, en donde todos los ítems considerados tienen una correlación ítem-test mayor.20. Ver Tabla 1.

Tabla 1. Especificaciones de los registros de observación de conductas de emprendimiento (ROCE- 2010).

Dimensión

\begin{tabular}{|c|c|}
\hline $\begin{array}{l}\text { Disposición de la } \\
\text { oportunidad }\end{array}$ & $\begin{array}{l}\text { a. En la fecha prefijada los comuneros nos esperan en el lugar acordado. } \\
\text { b. Los participantes se organizan para realizar una actividad productiva } \\
\text { (chalina) paralela a la propia de la comunidad. } \\
\text { c. Toman la iniciativa de evaluar su capacidad para cada actividad } \\
\text { propuesta. }\end{array}$ \\
\hline $\begin{array}{l}\text { Aprovechamiento } \\
\text { y responsabilidad } \\
\text { del recurso } \\
\text { económico y } \\
\text { humano }\end{array}$ & $\begin{array}{l}\text { a. El/la participante solicita que la organización incluya nuevos } \\
\text { productos porque pueden trabajarlo o realizarlo. } \\
\text { b. Se distribuyen la materia prima (lana) según requerimiento del diseño } \\
\text { del producto. } \\
\text { c. Usan la materia prima que se han distribuido y lo que sobra lo } \\
\text { devuelven. } \\
\text { d. Se distribuyen las tareas según sus habilidades y competencias para la } \\
\text { actividad productiva del taller (chalina). } \\
\text { e. Asisten puntualmente a los talleres de capacitación y a las asambleas } \\
\text { de la actividad productiva. } \\
\text { f. Reconocen las competencias y habilidades para la realización de la } \\
\text { actividad de sus compañeras (os). }\end{array}$ \\
\hline $\begin{array}{l}\text { Disposición al } \\
\text { riesgo }\end{array}$ & $\begin{array}{l}\text { a. Emprende un negocio conociendo el nivel de inversión que realiza. } \\
\text { b. Se informa previamente de lo que se arriesga en un negocio que } \\
\text { emprende. }\end{array}$ \\
\hline $\begin{array}{l}\text { Estabilidad y } \\
\text { serenidad en } \\
\text { incertidumbre }\end{array}$ & $\begin{array}{l}\text { a. Ejecutan sus decisiones cueste lo que les cueste (dejan de hacer sus } \\
\text { labores cotidianas). } \\
\text { b. Hay expresión de alegría en la ejecución de sus tareas. } \\
\text { c. Expresan su descontento para lograr alternativas de solución. } \\
\text { d. Presentan nuevos productos de Expresión Cultural. }\end{array}$ \\
\hline $\begin{array}{l}\text { Disposición a la } \\
\text { Innovación }\end{array}$ & $\begin{array}{l}\text { a. En el diseño de sus expresiones culturales adicionan nuevos } \\
\text { contenidos. } \\
\text { b. Identifican nuevas líneas de producción. } \\
\text { c. Expresan alegría cuando les compran los productos que vienen } \\
\text { confeccionando. } \\
\text { d. Comunican que usan o lo que emplean los productos que vienen } \\
\text { confeccionando. }\end{array}$ \\
\hline $\begin{array}{l}\text { Administración } \\
\text { del caos }\end{array}$ & $\begin{array}{l}\text { a. Los participantes se organizan para producir aún, cuando no tienen } \\
\text { claridad de lo que van a hacer. } \\
\text { b. Los participantes vencen la adversidad del idioma cuando se } \\
\text { relacionan con foráneos. } \\
\text { c. Los participantes aceptan el cambio o renovación de comisiones. } \\
\text { d. Estan organizados en equipos; distribuyen la información. } \\
\text { e. Los participantes toman decisiones sin esperar la aprobación de la } \\
\text { asamblea. }\end{array}$ \\
\hline
\end{tabular}




\section{Escala de actitudes hacia el emprendimiento (EAE-2010)}

La escala de actitudes hacia el emprendimiento con confiabilidad es de 0,83 por consistencia interna. La validez por correlación ítem total; el 100\% de ítems presenta correlación adecuada ítem total; construida en base a la Tabla 2 .

Para la escala de actitudes hacia el emprendimiento de la población rural, se consideró la validez de contenido realizada mediante criterio de jueces, para esto se solicitó su apreciación sobre la pertinencia de los reactivos. Para la fiabilidad de las puntuaciones del test de actitudes se hizo un análisis de consistencia interna, obteniéndose un coeficiente $\alpha=.83$, en donde todos los ítems considerados tienen una correlación ítem-test mayor.20 (Se eliminaron los ítems 2, 6, 7, 11, 14, 15, 24 iniciales por no satisfacer esta condición).

Tabla 2. Especificaciones: Actitudes hacia el Emprendimiento

Dimensión

Disposición de la Oportunidad

\section{Disposición de la Oportunidad}

\begin{tabular}{l} 
Disposición a la Innovación \\
\hline Disposición al riesgo \\
\hline $\begin{array}{l}\text { Aprovechamiento y responsabilidad del } \\
\text { recurso económico y humano }\end{array}$
\end{tabular}

Estabilidad y serenidad en incertidumbre
Identificación de oportunidades.

Aprovechamiento de la oportunidad.

Efectivizar en operaciones la oportunidad.

Gestionar las operaciones en procesos sus tareas.

\section{Iniciativa.}

Adicionar valor agregado a sus recursos.

Conocimiento gustos del mercado.

Vencer el temor al fracaso.

Audacia.

Visión de futuro.

Visualización de la utilidad costo beneficio.

Valorización de recursos humanos.

Valorización de expresiones culturales.

Valorización de su paisaje geográfico y ambiental.

Tomar decisiones.

Flexibilidad.

Respuesta creativa observada en productos.

Administración del caos

Desafío.

Organización flexible.

Organización en empoderamiento.

\section{FOCUS GROUP O GRUPOS DE ENFOQUE}

El proceso grupal de la dinámica de grupo se centra básicamente en el modelo interaccionista de Robert Bales, de manera simplificada aplicado el proceso grupal en cuatro etapas: 
a. Presentación: Preguntas y respuestas.

b. Producción: El contenido del taller, con técnicas lúdicas en base a las tareas de su tradicional actividad productiva, para el caso el tejido a palitos.

c. Análisis y generalización dirigida a su vida productiva, como por ejemplo el de logro de utilidades que puedan lograr a partir su actividad para su contexto social.

d. Aplicación al contexto de su vida cotidiana y comercial.

El contenido de cada sesión tiene por objetivo la transmisión de un concepto, el mismo será identificado por los participantes durante la realización de las tareas de la actividad laboral, para luego ser empleado en la próxima sesión en la etapa de producción.

Previamente, en cada sesión, hay un periodo o etapa de vinculación entre los investigadores con los participantes mediante preguntas y respuestas sobre el motivo o temática de la sesión, como por ejemplo una de las Prof. de la I.E. inicia la sesión explicando a las colaboradoras el objetivo organizar la unidad productiva; ya que las actividades tuvieron el objetivo de vincular los términos de comercialización y canal de ventas, con feria. Se llevó a cabo dos talleres de dinámica de grupo, los mismos tuvo como el proceso de transferencia la exposición de la Feria Municipal de Caraz, con su propio stand

Las cuatro etapas del proceso de la dinámica de grupo se llevan a cabo durante cada sesión del taller, se puede iniciar la primera etapa preguntando cómo han cumplido sus acuerdos de la última sesión. En la etapa de producción para el logro de aprendizaje del concepto de economía se emplea como estímulo tangible, por ejemplo la "Ficha del empleo de términos básicos económicos para la contabilidad". Aun cuando el contenido es abstracto, les facilita percibir la evidencia de sus resultados económicos en la distribución del dinero obtenido de manera concreta por su gestión, posibilitando su comprensión y descripción, es resaltante la participación de las colaboradoras a pesar que las no letradas no lo leían pero observando a las que leen mostraron su aceptación, y manifestando su opinión y su proyección para su agrupación.

De ahí que, la Ficha (Figura 1) tuviera gran acogida así como la presencia del dinero procedente de la venta de las chalinas, con sorpresa por estos réditos; porque ya concebían al papel escrito como un instrumento de control de sus resultados. Producción que, por la manera de haber sido presentados sus beneficios, les generó alegría y la disponibilidad para continuar; dando paso a que las colaboradoras pudieran transferir el mismo proceso a otros productos propios como la arveja. Por tratarse de dinero estuvo presente el Teniente Gobernador, quien también estuvo entusiasta y se preguntó “¿se puede hacer también con nuestros otros productos?”, respondiendo al unísono "catay" (por supuesto). 
Programa de Alfabetización Económica sobre Conductas de Emprendimiento en Pobladores Quechua Hablantes...

COMUNIDAD CAMPESINA CRUZ DE MAYO - Caserío de Queral

Periodo: Noviembre 2010 / Tipo de Producto: Chalina

\begin{tabular}{|c|c|c|c|c|c|c|}
\hline \multirow[b]{2}{*}{$\mathbf{N}^{\circ}$} & \multirow{2}{*}{$\begin{array}{c}\text { Nombres y } \\
\text { Apellidos }\end{array}$} & \multirow[b]{2}{*}{ Productos } & \multicolumn{3}{|c|}{ COSTO } & Firma \\
\hline & & & $\begin{array}{c}\text { Materia } \\
\text { prima }\end{array}$ & $\begin{array}{c}\text { Mano } \\
\text { de obra }\end{array}$ & $\begin{array}{l}\text { Aporte a } \\
\text { asociación }\end{array}$ & Precio \\
\hline \multirow{3}{*}{1} & \multirow{3}{*}{$\begin{array}{l}\text { Carmen Dalila } \\
\text { Lourdes }\end{array}$} & Bufanda verde & 5.00 & 10.00 & 10.00 & 25.00 \\
\hline & & Bufanda marrón & 5.00 & 10.00 & 10.00 & 25.00 \\
\hline & & Bufanda blanca & 5.00 & 10.00 & 10.00 & 25.00 \\
\hline 2 & Alicia & Bufanda rosada & 5.00 & 10.00 & 10.00 & $25.00 *$ \\
\hline 3 & Alicia Norma & Bufanda verde limón & 5.00 & 10.00 & 10.00 & 25.00 \\
\hline \multirow[t]{2}{*}{4} & Rosa & Bufanda celeste & 5.00 & 10.00 & & $25.00 *$ \\
\hline & TOTAL & & 20.00 & 40.00 & 40.00 & 100.00 \\
\hline \multicolumn{3}{|c|}{ Productos vendidos $=4$} & ndidos $=$ & & \multicolumn{2}{|c|}{ TOTAL $=S / .100,00$} \\
\hline \multicolumn{3}{|c|}{ Carmen } & & & \multicolumn{2}{|c|}{$\begin{array}{c}\text { Manuel } \\
\text { Teniente Gobernador }\end{array}$} \\
\hline
\end{tabular}

Figura 1. Ficha del empleo de términos básicos y económicos para la contabilidad.

Correspondiente a la etapa de análisis y generalización de la dinámica de grupo, se observa con la descripción del término o vocablo comparándolo con la acción que desempeñan, por ejemplo "el costo para dar precio".

La cuarta etapa del proceso grupal, se dio con la expresión del Teniente Gobernador, a propósito de la dinámica de grupo de la distribución de ganancias.

\section{RESULTADOS}

\section{Resultados descriptivos y comparativos}

Las actitudes hacia el emprendimiento son positivas, a nivel promedio con tendencia alta (grado 3) en la escala de 4 grados. Tabla 3; tanto del grupo experimental, colaboradoras del caserío Queral, y del grupo de control comparativo de padres de familia del 3er grado de la I. E. 2 de Mayo de Caraz.

Tabla 3. Total del nivel de las actitudes hacia el emprendimiento

\begin{tabular}{lccccc}
\hline \multirow{2}{*}{ Dimensión } & \multicolumn{2}{c}{ Queral } & & \multicolumn{2}{c}{ 2 de Mayo } \\
\cline { 2 - 3 } & $M$ & $D E$ & & $M$ & $D E$ \\
\hline Disposición a la innovación & 2.96 & 0.34 & & 3.21 & 0.38 \\
\hline Disposición al riesgo & 3.00 & 0.51 & 3.11 & 0.49 \\
\hline $\begin{array}{l}\text { Aprovechamiento y responsabilidad del recurso económico } \\
\text { y humano }\end{array}$ & 2.91 & 0.45 & 3.14 & 0.41 \\
\hline Administración del caos & Total & 2.79 & 0.35 & 3.27 & 0.44 \\
\hline
\end{tabular}

Muestra: 33 sujetos 
Evaluación antes y después de la aplicación del programa de alfabetización económica sobre conductas de emprendimiento en pobladores quechuas hablantes de Caseríos de Caraz

Inicialmente se realizó un análisis de la normalidad con el estadístico de Kolgomorov Smirnov, con corrección de Lilliefors, para cada uno de los grupos que conforman el grupo experimental, de control y de la diferencias de las puntuaciones totales antes y después de la intervención, obteniéndose en ellos valores de significancia $\mathrm{p}<.05$, por lo que para el análisis inferencial se usará estadísticos no paramétricos.

Con respecto a la comparación de grupos relacionados antes y después de la intervención se tiene diferencias estadísticamente significativas entre las evaluaciones antes y después en las conductas de emprendimiento en el grupo experimental de Queral, esto realizado con la prueba de rangos con signo de Wilcoxon ( $\mathrm{Z}=$ $-2.812, \mathrm{p}=.005)$. Para verificar que las diferencias observadas en el grupo experimental se debe, básicamente, a la intervención, donde se analiza la diferencia de las puntuaciones antes y después del grupo de control en donde no se rechaza la hipótesis nula de igualdad de las puntuaciones antes y después $(Z=-0.135$, $\mathrm{p}=.892$ ), ver Tabla 4 .

Además, se realizó la medida del tamaño del efecto, la que a definición de Cohen (1988) es el grado en el que un fenómeno está presente en la población. Para el mismo se usó la fórmula de la diferencia estandarizada de Cohen para el caso de heterocedastidad, en el que se considera la mediana en lugar de la media, al ser el primero una mejor medida de tendencia central en distribuciones no paramétricas. Se obtiene $\mathrm{d}=1.48$ para el caso de grupo experimental y $\mathrm{d}=0.18$ para el grupo de control, lo que a convención de Cohen corresponde el primero a un efecto grande y el segundo valor se encuentra por debajo de un efecto pequeño.

Tabla 4. Análisis inferencial de las conductas de emprendimiento antes y después de la intervención

\begin{tabular}{|c|c|c|c|c|c|c|c|}
\hline & \multicolumn{2}{|c|}{ Evaluación antes } & \multicolumn{2}{|c|}{$\begin{array}{c}\text { Evaluación } \\
\text { después }\end{array}$} & \multicolumn{2}{|c|}{$\begin{array}{l}\text { Prueba de } \\
\text { Wilcoxon }\end{array}$} & \multirow{2}{*}{$\begin{array}{c}\text { Tamaño } \\
\text { del efecto } \\
\text { d }\end{array}$} \\
\hline & M & $\mathrm{DE}$ & M & $\mathrm{DE}$ & $\mathrm{Z}$ & $\mathrm{p}$ & \\
\hline $\begin{array}{l}\text { Grupo experimental } \\
(\text { Queral, } \mathrm{n}=10)\end{array}$ & 11.40 & 4.09 & 16.60 & 3.31 & -2.814 & .005 & 1.48 \\
\hline $\begin{array}{l}\text { Grupo de control }(2 \mathrm{de} \\
\text { mayo, } \mathrm{n}=7)\end{array}$ & 17.29 & 7.13 & 19.14 & 3.67 & -0.315 & .752 & 0.18 \\
\hline
\end{tabular}

Los colaboradores han expuesto en la Feria Municipal 2010 la producción de las chalinas, manifestado y demostrado en su comportamiento que dicha expresión cultural, es un bien a ser producto comercial y una alternativa para mejorar la pobre economía. Motivación en la implementación de la constitución y desarrollo 
de la Asociación de Productores del Caserío de Queral Tuco Wain Perú; alternativa factible y enriquecedora del recurso humano y capital social de la Comunidad a propósito de reducir la brecha de desigualdad en la distribución, PNUD (2010) y en concordancia a los lineamientos y recomendaciones que cumplen las chalinas como bienes culturales planteados por la Unesco (2005).

El involucrarse y la participación, como conductas organizacionales son observadas, como resultado del taller de capacitación a propósito del término de Marca, la decisión del símbolo que represente a su asociación, el "Tuco", elegido en un taller previo entre dos posibles símbolos: El Tuco y Margaritas, propuestas mediante la "lluvia de ideas" en un espacio físico al aire libre, sentadas en el suelo, dando varias alternativas entre bromas y picardía, empero expresaron entre risas que no sabían por qué, tema a seguir investigándose, en referencia verbal de la Dra. Angélica Aranguren Paz, está relacionado con la dualidad del pensamiento andino "el bien para quien posee el Tucu y el mal para quien lo desea".

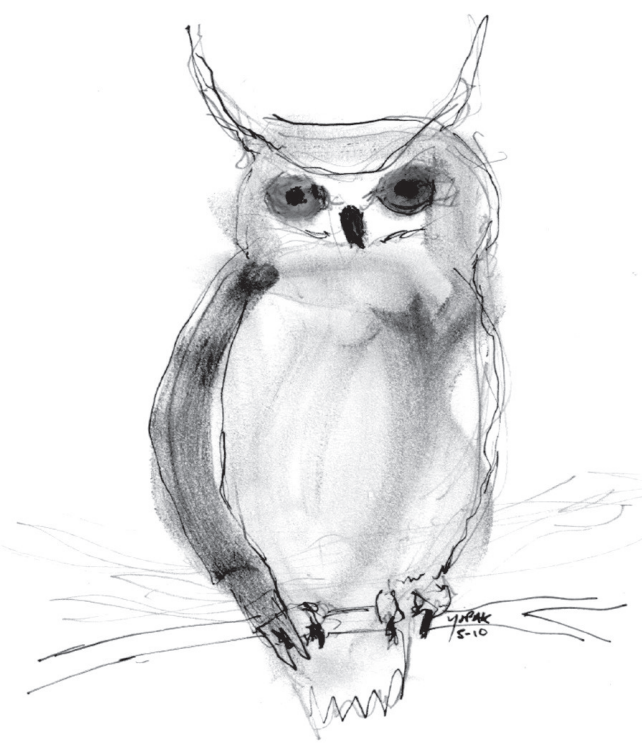

Figura 2: Símbolo de marca "El Tuco" de Asociación de productores del caserío de Queral-Tuco Wain-Perú. Dibujo original Autor: Yurak Britto Vivas lunem3@hotmail.com

La conducta de "aprovechar la oportunidad" no se manifiesta cuando se trata de desarrollar actividades propias de capacitación, en especial con tareas académicas, abstractas o en aquellas donde sus habilidades no son apropias para las tareas respectivas. Así por ejemplo en el taller de bisutería desarrollado en la comunidad, con el objetivo de precisar los términos básicos de economía: costos, comercialización y gestión; la asistencia fue de las jóvenes y para las tareas de corte confección de los jóvenes, no así los adultos quienes no se presentaron a dichos talleres. 
Las conductas de aprovechamiento y responsabilidad del recurso económico y humano, se hacen evidentes en la distribución que hacen de la lana, donde el taller tuvo el objetivo de transmitir términos como insumos y materia prima. El espacio de la dinámica de grupo permitió observar la responsabilidad en el manejo del limitado recurso y la asunción de roles de acuerdo a sus habilidades, y el uso del mismo durante el taller de distribución de ingresos.

Asumir el riesgo no está dentro de los cálculos en el comportamiento de las colaboradoras del estudio, pues si bien se les observa entusiastas ante nuevas propuestas, al momento de llevar a cabo las acciones que demanda tomar decisión se abstienen o no asisten. Destaca que mantienen su patrón y normas en la elaboración de sus productos, logran aceptar el empleo de colores que no son frecuentes o no empleados en la confección de las chalinas, pero mantienen sus líneas y diagramas, diseños que le dan la peculiaridad y calidad a sus productos, de ahí que solo se observa la Innovación, en cuanto a la forma más que en el contenido, caracterizando la permanencia de su cultura.

Se infiere la estabilidad y serenidad en su vida cotidiana y sus retos, aun cuando hay mucha inquietud y desazón por la carencia del agua. Ante dicho recurso podemos observar que administran con pulcritud y distribuyen con equidad el cumplimento de las cuotas asignadas por el comité respectivo, más no podemos inferir que puedan tenerlo para los negocios, ¿cómo es su postura ante la incertidumbre?, es una conducta individual más que corporativa o asociativa para emprender.

En la administración del caos, son perseverantes y logran salir ante la adversidad y conflicto, organizándose y asumiendo sus roles de acuerdo a lo acordado en su asamblea, cumplen de manera sacrificada cuando se trata de la comunidad. Descuidando cuando se trata de hacer negocios conjuntos, dudan entre ellos, manifiestan con audacia que son unos "filibusteros" calificativo que en ocasiones se le atribuye cuando no dejan su negocio para cumplir con su palabra (fuera del contexto de asamblea o de un acuerdo en ella) o compromisos asumidos, porque arguyen razones poco fiables, o evidencian dudas o mofa por su actuación. Su presencia a los talleres lo han postergado por sus ocupaciones de su vida cotidiana, pero cuando se ha tratado de aquellas tareas (bisutería, corte confesión) para los grupos respectivos que asistieron, manteniéndose hasta concluir su producto.

Para identificar los intereses del grupo de control conformado por colaboradoras quechua hablantes urbanas, a quienes no se aplicó la variable independiente, pero se les proporcionó un taller sobre bisutería, luego de explorar sus intereses y en concordancia con ellas, con el objetivo de retribuir su colaboración en el proyecto se: 


\section{Diagnóstico de necesidades de capacitación: taller de alfabetización económica}

Población: Padres de familia colaboradores de la I. E. 2 de Mayo, 3er. Grado. $\mathrm{Al}$ inicio del trabajo con el grupo de control, se realizo el siguiente diagnóstico, Tabla 5

Tabla 5. Necesidades y expectativas del grupo de control

\section{Mis expectativas}

1. Estamos seguros que obtendremos buenos resultados del taller.

2. Aprender para poder enseñar y ayudar a mi hogar económicamente.

3. Ayuda de los psicólogos para los niños.

4. Aprenderá ayudar a mi hogar y a otras personas económicamente.

5. Necesito ayuda psicológica.

6. Sería bueno el proyecto del taller de bisutería.

7. Necesito el taller de la bisutería.

8. Necesito que nos apoyen psicológicamente a mí y toda mi familia.

9. Capacitación - economía.

10. Aprender ayudar, para ayudar a otros.

11. Apoye a su hijo en el tratamiento psicológico.

12. Quiero que sus hijos sean los mejores del salón.

13. Quiero que su hijo aprenda.

14. Quiero que mi hijo sea el mejor en el salón.

\section{Mis expectativas para ayudar al aula de clase}

1. Que el salón este más implementado.

2. Que el aula tenga algunos cuadros.

3. Que el aula este implementado.

4. Pintar el aula y adornar.

5. Apoyar en todo para dar buena imagen.

6. Que el aula tenga todas las comodidades necesarias.

7. Ambientación del aula con sus respectivos sectores.

8. Decorar el aula en base a la bisutería que elaboramos.

9. Tener orden y responsabilidad.

10. Implementar botiquín.

11. Ayuda a los niños a mejorar en sus estudios.

12. Para que su hijo aprenda.

13. Debemos mejorar el aula.

\section{Mis Expectativas del taller}

1. Aprender como formar una empresa.

2. Quisiera obtener buenos resultados y tengo fe que la lograremos.

3. Estamos dispuestos a poner todo de lo nuestro.

4. Aprender y dar todo de nosotras.

5. Pintura.

6. Reparar carpetas.

7. Que sea lo máximo.

8. Que de seguro será un gran avance.

9. Trabajar con los hijos haciendo collares.

10. Tenemos fe que nos enseñaran bien y lo lograremos.

11. Queremos muebles con mesa.

12. Trabajar junto con nuestros hijos y aprendamos poniendo de nuestro a parte.

13. Tenemos fe que nos enseñarán bien y lograremos lo mejor.

14. El tejido.

15. Cosas, collares, bordado, tejido para hacer pulseras.
Mis expectativas de los padres de familia

1. Que los padres sean participativos en el proyecto.

2. Que los padres cumplan con todo lo que se le pida.

3. Que los padres sean más puntuales.

4. Qué los padres cumplan con lo acordado.

5. Yo desearía la unión de los padres.

6. Que los padres sean puntuales.

7. Que el profesor no deba hablar con celular en el momento de clases.

8. Que todos asistamos, no falten y nos apoyemos siempre.

9. Mucha responsabilidad de los padres y que aprovechen esta oportunidad que se les brindan con mucha voluntad.

10. Que todos los padres seamos unidos y nos apoyamos mutuamente.

11. Participen todos los padres en reunión.

12. Que los padres participemos todos unidos.

13. Apoyen a sus hijos.

14. Que todos los padres debamos ser responsables.

Quienes priorizaron el adiestramiento en bisutería, el que fue otorgado. 


\section{Resultados y discusión}

La muestra del grupo experimental estuvo constituida por las colaboradoras de Queral que de la producción de chalinas, descrita por ellas como una entre los bienes o servicios de expresión cultural a ser nominados productos comerciales, según los lineamientos y recomendaciones por la Unesco (2005) como alternativa de mejorar la pobre economía y posibilitar el inicio en su implementación de la constitución y desarrollo de la "Asociación de productores del caserío de Queral Tuco - Wain - Perú"; alternativa factible y enriquecedora del recurso humano y capital social de la comunidad, a propósito de reducir la brecha de desigualdad en la distribución de recursos, PNUD (2010), de manera sostenible.

La respuesta de emprendimiento, con un porcentaje mayor al promedio, se dio en el taller referido a la distribución de la utilidad en la venta de las chalinas, usando el formato donde figuraban los términos básicos de economía: Materia prima, mano de obra, Aporte a la asociación, precio, con el indicador dinero (Resultados: 1er. momento S/. 192 y en un 2do. momento S/.100 nuevos soles). Las ganancias se distribuyó entre las colaboradoras, según los términos señalados.

Demostraron alta actividad, "como salir de una incredulidad" y expresar beneplácito por el conocimiento sobre sus costos para poder "transferir" para el manejo de sus productos agrícolas. Su producción en este taller en relación a los anteriores y posteriores fue destacada, lo que confirma que el objetivo genera actividad, acción y disposición para su logro.

El involucrarse y la participación, como conductas organizacionales, observadas a propósito del término de Marca, o por la elección del "Tuco" como símbolo de su Asociación, grafica la importancia que tiene tomar decisión, un indicador de madurez y compromiso por la pertenencia.

Es importante señalar la discusión sobre el resultado encontrado donde no se dio diferencia en la conducta de emprendimiento antes y después en el grupo de control, se asume a que dicho grupo no se constituyó en una organización económica (empresa), a pesar que se organizaron y obtuvieron créditos o ganancias en la elaboración de bisutería para adquirir un estante para el aula. Puede haberse dado la conducta de emprendimiento personal pero no como unidad productiva como lo es, el Caserío de Queral.

La diferencia en ambos grupos se le puede atribuir a que la socialización económica posibilita la comprensión del mundo económico, el que requiere que el individuo construya una visión sistémica del modelo económico social en que está inserto, para desarrollar competencias y actitudes que le posibiliten un uso adecuado de sus recursos económicos, incluyendo los hábitos y conductas de consumo racionales y eficientes, que se han venido implementando desde 2004 en el grupo experimental. 


\section{CONCLUSIONES}

El Programa de alfabetización económica afecta las conductas de emprendimiento en los siguientes aspectos:

1. En el manejo de los recursos económicos y humanos.

2. Disposición a la Innovación.

3. Aprovechamiento de la oportunidad.

4. Toma de decisiones en condiciones de incertidumbre.

5. Administración del caos.

6. Son perseverantes y logran salir ante la adversidad y conflicto, organizándose y asumiendo sus roles a lo acordado en la asamblea de la comunidad.

7. Actitudes hacia el empoderamiento con tendencia positiva.

\section{IMPACTO}

Formación de una unidad productiva: Asociación de productores del caserío de Queral - Tuco - Wain - Perú

\section{RECOMENDACIONES}

Continuar con el seguimiento y realizar la transferencia para las otras actividades económicas, la agricultura: cultivos de alverja. Aplicar la metodología desarrollada a otras poblaciones, de las diferentes regiones, rurales en el interior del país y las zonas urbanas marginales.

\section{RECONOCIMIENTOS}

Instituto de Investigaciones Psicológicas de la Facultad de Psicología; Municipalidad Provincial de Huaylas Caraz; Prof. Víctor Laguna, por la traducción de la Escala Castellano - Quechua; las colaboradoras del Caserío de Queral y del I. E. 2 de Mayo Caraz del nivel primario aula 3er. sección A, profesor Martín Dongo; Profesores de la I.E. de Queral, en especial a las profesoras Olga Sáenz Polo y Elizabeth Emma Jáuregui López, por haber sido el nexo directo con las mujeres que crearon la asociación Tuco - Wain, en la traducción en los talleres quechuaespañol- quechua; Cetpro Francisco García Calderón de Pueblo Libre, desarrollo del taller de bisutería.; Ronald Castillo por el procedimiento estadístico; Roberto Aliaga por formateo y diseño de ayudas visuales para los talleres y Feria Municipal de Caraz.

\section{REFERENCIAS BIBLIOGRÁFICAS}

Ardila, R. (1993). Psicología Social de la Pobreza. En Whittakker, J. O. La Psicología Social en el Mundo de Hoy. (399-418) Edit. Trillas. 2da. Ed.

Blanchard, K.; Carlos J., y Randolph, A. (2002). Las 3 claves para el empowerment. Cómo dar poder para liberar la capacidad de las personas y lograr que den lo mejor de sí. Barcelona: Ediciones Granica. 
Bregman, P. (2009, 31 de Agosto). Cómo contrarrestar la resistencia al cambio. Harvard Business Review América Latina, Obtenido el 19 de Noviembre de 2010, de http:// www.hbral.com/blog/blog.asp?modulo $=2 \&$ idBlog $=35$.

Denegri, M.; Fernández, F.; Mireya I. y Ripio, M. (1999). Consumir para vivir y no vivir para consumir. Temuco: Ediciones Universidad de La Frontera.

Paredes M.; Hernandez, H.; Vicuña, L. y De Miguel, J Ma. (2007). Percepción del conocimiento de la demanda de productos y percepción hacia la interdependencia económica en el Caserío Queral, Caraz, Ancash, Revista de Investigación en Psicología, Vol. 10 No 1:109-124. Facultad de Psicología. UNMSM.

Paredes M.; Hernández, H; Vicuña L.; Rivera, J C. y Arias V. (2009). Percepción del conocimiento de las expresiones culturales y su revalorización integral en dos comunidades altoandinas en Ancash, Lima, Perú. Revista de Investigación en Psicología Vol. 12, No 2, 51-73. Facultad de Psicología. UNMSM.

Paredes, M. y De Miguel, J. Ma . (2004). Construcción Social de la Comunidad para el Desarrollo del Callejón de Huaylas, Caraz. Revista de Investigación en Psicología Vol. $7 \mathrm{~N}^{\mathrm{o}}$ 1, 81-102. Facultad de Psicología. UNMSM.

Paredes, M.; De Miguel, J. Ma.; Vicuña, L. y Hernández, H. (2005). El papel de la construcción social de la organización con respecto a la percepción de las condiciones de trabajo y el manejo del medio ambiente en 4 caseríos de una comunidad campesina andina - Caraz, Ancash. Revista del Instituto de Investigación Vol.8 N ${ }^{0} 16$ 57-67. Facultad de Ingeniería Geológica, Minería, Metalúrgica y Geografía, UNMSM.

Paredes, T. M. (2009). El Caserío es la unidad productiva en la articulación organizacional de zonas rurales altoandinas, Callejón de Huaylas, Ancash. Revista del Instituto de Investigaciones Económicas, Pensamiento Crítico, Nº 11:73-96, Junio, Lima, Perú. Economía, industria y riesgo. Facultad de Economía, UNMSM.

Piaget, Jean (1971). Psicología y Epistemología. Barcelona: Ediciones ARIEL S.A., Esplugues Llobregat Traducción castellana de Francisco J. Fernández Buey.

PNUD (2010). Informe sobre Desarrollo Humano 2010, La verdadera riqueza de las naciones: Caminos al desarrollo humano: La verdadera riqueza de las naciones. Edición del Vigésimo Aniversario.

United National Educational, Scientif and Cultural Organization (UNESCO) (2005). Documentos Fundamentales para el Patrimonio Cultural. Convención sobre la Protección y Promoción de la Diversidad de las Expresiones Culturales. 117-130. París. http://www.cultura.gob.pe/sites/default/files/archivosadjuntos/2013/05/ iiidocumentosfundamentales.pdf

United National Educational, Scientif and Cultural Organization (UNESCO) (2008). Informe de seguimiento de la educación en el mundo "Superar la desigualdad: por qué es importante la gobernanza”. http://unesdoc.unesco.org/images/0017/001776/177609s.pdf

Webb, R. (2012). Las zonas rurales del país crecen en 5\% al año. El Comercio, Economía,Pg. B7 del día miércoles 27 de junio del 2012. 